\title{
Preparation and Performance of Warm Mix Colored Asphalt Modified by Oxidized Polyethylene Wax
}

\author{
Xinde Tang ${ }^{1, a}$, Xuxiang $\mathrm{Tan}^{1}$, Xiuying $\mathrm{Hu}^{1}$, Yiran $\mathrm{Hu}^{1}$, Chunliang $\mathrm{Liu}^{1}$, \\ Chao Kong ${ }^{2}$ and Ying $\mathrm{Li}^{2}$ \\ ${ }^{1}$ School of Material Science and Engineering, Shandong Jiaotong University, Jinan 250357. China \\ ${ }^{2}$ Shandong High-Speed Material Storage and Transportation Co., Ltd., Zibo 255414, China \\ axdtang8033@163.com
}

\begin{abstract}
Keywords: Warm mix colored asphalt; colored asphalt cement; oxidized polyethylene wax; physical performance.

Abstract. Colored asphalt cement was prepared by mixing aromatic oil with petroleum resin and polymer additives. Then the colored asphalt cement was modified by oxidized polyethylene (OPE) wax, resulting in warm mix colored asphalt cement. The content effect and mechanism of OPE wax on the performance of the warm mix colored asphalt cement were investigated.
\end{abstract}

\section{Introduction}

Black petroleum asphalt has been widely used in pavement construction [1,2]. With the development of economy and society, hardscape projects including asphalt driveways, asphalt walkways, asphalt streets, asphalt bike paths, and asphalt walking paths have been required colored pavement. Colored pavement as the role of urban traffic organization and landscape is increasingly concerned by people [3]. The colored pavement technology has made roads and the surrounding environment more coordinated, which is more conducive to beautify city and induce traffic. For example, different colored pavement in different lane is superior to the traffic signs line for traffic guidance, and the large area of color distinction gives directly visual guide for drivers, which played an important role of traffic organization [4,5]. To be suitable for facile construction and special application, further modification on colored asphalt could be realized by functional additives, such as inorganic nano material [6,7], coupling agent [8], warm mix additives [9], or anti-rutting agent [10].

The warm mix colored asphalt using the Sasobit and calcium stearate as warm mix additive was reported in previous research [9]. Compared to the traditional hot mix technique, the viscosity of warm mix asphalt can be reduced at elevated temperature by addition of warm mix additive. Furthermore, warm mix technique can save construction cost and decrease harmful emissions with better energy conservation, and economic and environmental benefit. Therefore, the development of warm mix additives for colored asphalt is of great significance.

Oxidized polyethylene (OPE) wax is an excellent polar wax with a certain amount of carbonyl and hydroxyl groups on the molecular chains. The compatibility of OPE with filler, pigment, or polar resin improves significantly, and the wettability and dispersion in polar system exhibit better than that of polyethylene wax. In addition, OPE possesses coupling performance. In this paper, The effect and mechanism of OPE wax on the performance of the warm mix colored asphalt cement were investigated.

\section{Experimental}

Materials. Aromatic oil, petroleum resins, and polymer additives such as ethylene vinyl acetate (EVA) copolymer and styrene-butadiene-styrene (SBS) block copolymer were purchased from Jinan Double Pavilion Chem. Co., Ltd, China. Oxidized polyethylene (OPE) wax was kindly offered by Weifang Sunny New Material Co., Ltd, China.

Preparation and physical performance of colored asphalt cement. A certain amount of aromatic oil, petroleum resin, and EVA were mixed and stirred at $150-160{ }^{\circ} \mathrm{C}$, then a small certain 
amount of SBS was added to the mixture under high-speed shearing at $170-180{ }^{\circ} \mathrm{C}$. By a developing procedure for $30 \mathrm{~min}$, colored asphalt cement was obtained.

Modification of OPE wax on colored asphalt cement. A series of different percent of OPE wax were added to the prepared colored asphalt cement under high-speed shearing at $170-180{ }^{\circ} \mathrm{C}$. The warm mix colored asphalt cements were obtained.

Performance test of colored asphalt cement and warm mix colored asphalt cement. The physical performance test, including penetration degree, ductility, and softing point were conducted according to Test Procedures for Asphalt and Asphalt Mixtures for Highway Engineering (T0604-2000, T0605-2000, and T0606-2000) .

\section{Results and Discussion}

Preparation of colored asphalt cement. The combination of aromatic oil with petroleum resin, polymer additives can simulate common petroleum asphalt. The physical performance of the resulting corlored asphalt cement before and after aging were show in Table 1. Before aging, the physical performance of sample 2 and 3 is similar to that of 70\# petroleum asphalt, while sample 1 similar with 50\# petroleum asphalt. After aging, the penetration degree and ductility of the colored asphalt cement decreased as expected. The mass loss and flash point after aging are accordance with the actual requirements. Sample 2 and 3 were chosen for further modification in the following procedure.

Table 1 Physcial performance of colored asphalt cement before and after aging

\begin{tabular}{|c|ccc|cccc|}
\hline \multirow{3}{*}{ Sample } & \multicolumn{3}{|c|}{ Before aging } & \multicolumn{5}{c|}{ After aging } \\
& \multicolumn{2}{|c|}{ Penetration Degree Ductility Softing point } & Penetration Degree & Ductility Mass loss \\
& $\left(25^{\circ} \mathrm{C} / 0.1 \mathrm{~mm}\right)$ & $\left(10^{\circ} \mathrm{C} / \mathrm{cm}\right)$ & $\left({ }^{\circ} \mathrm{C}\right)$ & $\left(25{ }^{\circ} \mathrm{C} / 0.1 \mathrm{~mm}\right)$ & $\left(10^{\circ} \mathrm{C} / \mathrm{cm}\right)$ & $(\%)$ & $\left({ }^{\circ} \mathrm{C}\right)$ \\
\hline 1 & 51.0 & 56.0 & 55.8 & 41.6 & 40.8 & -0.85 & 230 \\
2 & 62.1 & 54.8 & 55.7 & 45.9 & 44.8 & -0.96 & 240 \\
3 & 61.2 & 65.2 & 55.6 & 44.3 & 46.0 & -0.95 & 238 \\
\hline
\end{tabular}

Modification of colored asphalt cement. OPE wax was applied to modify the colored asphalt cement in order to realize warm mix goal. OPE wax plays a crucial role in the modification of colored asphalt. As shown in Table 2, after addition of OPE wax, significant changes occurred in the physical performance for sample 2 . The penetration degree gradually dereased from 62.1 to 42.1, 32.4, 26.2, and 22.6 by $2 \%, 3 \%, 4 \%$, and $6 \%(\mathrm{~m} / \mathrm{m})$ addition of OPE wax, respectively. This indicated that the modified colored asphalt cement shows excellent rigidity by combination of OPE wax with colored asphalt cement, accordingly, which gives rise to an significant increased from the original $55.7^{\circ} \mathrm{C}$ to $62.8,81.0,98.0$, and $>100$ for softing point. In other word, the addition of OPE wax gives rise to excellent high temperature stability for colored asphalt cement, which implys that OPE wax has the potential for anti-rutting agent. Meanwhile, the ductility dereased with addition of OPE wax, which can be attributed to the bad compatibility between OPE wax and colored asphalt cement. After aging, the change tendency of penetration degree, ductility and softing point is accordance with that of before aging. In addition, the mass loss shows a rational derease, which can be ascribed to the fine anti-oxidation of OPE wax-modified colored asphalt cement compared to the original colored asphalt cement.

As shown in Table 3, OPE wax was also applied to modify the sample 3. With $3 \%(\mathrm{~m} / \mathrm{m})$ addition of OPE wax, the penetration degree and ductility dereased obviously, and the softing point increased significantly. Under the identical addition of OPE wax (3\%), compared to sample 2, sample 3 displays better low temperature stability, which can be attributed to the higher content of aromatic oil in the colored asphalt cement. This suggestes that the physical performance of warm mix colored 
asphalt cement may be controlled not only by changing the content of OPE wax, but also adjusting the component content.

Table 2 Modification of OPE wax on colored asphalt cement of sample 2

\begin{tabular}{|c|ccc|ccc|}
\hline \multirow{2}{*}{ Sample 2 } & \multicolumn{3}{|c|}{ Before aging } & \multicolumn{3}{c|}{ After aging } \\
& \multicolumn{2}{|c|}{ Penetration Degree Ductility Softing point } & \multicolumn{3}{c|}{ Penetration Degree Ductility Mass loss } \\
& $\left(25^{\circ} \mathrm{C} / 0.1 \mathrm{~mm}\right)$ & $\left(10^{\circ} \mathrm{C} / \mathrm{cm}\right)$ & $\left({ }^{\circ} \mathrm{C}\right)$ & $\left.55{ }^{\circ} \mathrm{C} / 0.1 \mathrm{~mm}\right)$ & $\left(10{ }^{\circ} \mathrm{C} / \mathrm{cm}\right)$ & $(\%)$ \\
\hline Original & 62.1 & 54.8 & 55.7 & 45.9 & 44.8 & -0.85 \\
$2 \%$ addition of OPE & 42.1 & 45.9 & 62.8 & 24.4 & 1.2 & -1.2 \\
$3 \%$ addition of OPE & 32.4 & 28.5 & 81.0 & 20.5 & 0.7 & -0.8 \\
$4 \%$ addition of OPE & 26.2 & 28.1 & 98.0 & 24.6 & 1.6 & -1.0 \\
$6 \%$ addition of OPE & 22.6 & 27.7 & $>100$ & 19.6 & 1.6 & -0.9 \\
\hline
\end{tabular}

Table 3 Modification of OPE wax on colored asphalt cement of sample 3

\begin{tabular}{|c|ccc|ccc|}
\hline \multirow{2}{*}{ Sample 3 } & \multicolumn{3}{|c|}{ Before aging } & \multicolumn{3}{c|}{ After aging } \\
& \multicolumn{2}{|c|}{ Penetration Degree Ductility Softing point } & \multicolumn{3}{c|}{ Penetration Degree Ductility Mass loss } \\
& $\left(25{ }^{\circ} \mathrm{C} / 0.1 \mathrm{~mm}\right)$ & $\left(10{ }^{\circ} \mathrm{C} / \mathrm{cm}\right)$ & $\left({ }^{\circ} \mathrm{C}\right)$ & $\left(25{ }^{\circ} \mathrm{C} / 0.1 \mathrm{~mm}\right)$ & $\left(10{ }^{\circ} \mathrm{C} / \mathrm{cm}\right)$ & $(\%)$ \\
\hline Original & 61.2 & 65.2 & 55.6 & 44.3 & 46.0 & -0.95 \\
$3 \%$ addition of OPE & 47.6 & 54.0 & 98.0 & 37.8 & 41.6 & -0.8 \\
\hline
\end{tabular}

Mechanism of wam mix colored aspalt cement. Warm mix asphalt (WMA) technique is based on the theory of interface chemistry. The WMA process can reduce the viscosity of asphalt at high temperature, accordingly can improve the fluidity of the asphalt mixture. The influence of the warm mix additive on asphalt is related to the type and content of the warm mix additive. Actually, OPE wax is a kind of surfactants, in which there are some polar groups such as carbonyl and hydroxyl on the molecular chains. Warm mix additive was applied by wet or dry process. In this paper, wet process was employed for the preparation of warm mix colored asphalt cement. During the mixture mixing process, warm mix colored asphalt cement and aggregates were added into the mixing pot. Under the mechanical force stirring, the addition of OPE wax not only increase the mixture mixing workability, but also prevent the colored asphalt mortar aggregation. During mixture paving and compaction process, OPE wax plays an important role and thus improves the paving speed mixture compaction, so as to realize the mixture warm mix, paving, and compaction.

\section{Summary}

Colored asphalt cements simulated the black petroleum asphalt were prepared by combination of aromatic oil with petroleum resin and functional polymers. Through the addition of different content of OPE wax, a series of warm mix colored asphalt cements were prepared. The physical performance indexes including penetration degree, ductility, and softing point of colored asphalt cement and warm mix colored asphalt cement were tested. With an increase of OPE wax content, the resulting warm mix colored asphalt displayed dereased penetration degree and ductility and increased softing point, which indicated OPE wax is beneficial to the high temperature stability and unfavourable to the low temperature stability. As an efficient warm mix additive, OPE wax can dramatically decrease mix temperature, save energy, and protect environment. In addition, OPE wax can act as a potential anti-rutting agent for colored asphalt.

Acknowledgements This work was financially supported by Shandong Provincial Science and Technology Development Plan Project of China (2014GGX102013), Traffic Application Basic 
Research Project of Ministry of Communications of China (2007319817090), Western Traffic Construction Project of China (200731800064).

\section{References}

[1] X.D. Tang, Z.G. He, W.J. Yun, Z. Li and Y. Liu: Petroleum Asphalt Vol. 22 (2008), p. 6

[2] X.D. Tang and N.F. Han: Mater. Rev. Vol. 22 (2008), p. 38

[3] X.G. Zhu, X.N. Zhang and H.J. Li: Sci. Tech. Engng. Vol. 10 (2010), p. 4548

[4] X.N. Zhang: City paving landscape (China Buiding Industry Press, Beijing 2000).

[5] G.L Zou and X.N. Zhang: Colored asphalt pavement technology research report (South China Univ. Tech, Guangzhou 2005).

[6] J. Yu, B. Li, X. Zeng, X.L. Wang and H.G. Xu: J. Wuhan Univ. Technol. Vol. 29 (2007), p. 65

[7] X.D. Tang, N. F. Han, Z.G. He, J.J. Li and D.L. Meng: J. Building Mater. Vol. 13 (2010), p. 550

[8] X.D. Tang, Z.G. He, J. Xu, J.J. Wang and T. Lin: Mater. Rev. Vol. 23 (2009), p. 406

[9] J.F. Gong. Shangqing. Vol. 4 (2011), p. 105

[10] H. Gao. J.X. Li and H.L. Luo. Petroleum Processing and Petrochemicals. Vol. 37 (2006), p. 56 